\title{
The effect of high fat dietary modification and nutritional status on the outcome of critically ill ventilated children: single-center study
}

Nehal Mohamed El Koofy, MD1', Hanaa Ibrahim Rady, MD', Shrouk Moataz Abdallah, MD', Hafez Mahmoud Bazaraa, MD', Walaa Ahmed Rabie, MD², Ahmed Ali El-Ayadi, MD'

Departments of 'Pediatrics, ${ }^{2}$ Clinical and Chemical Pathology, Faculty of Medicine, Cairo University, Egypt

Background: Ventilator dependency constitutes a major problem in the intensive care setting. Malnutrition is considered a major determinant of extubation failure, however, attention has been attracted to modulating carbon dioxide production through decreasing carbohydrate loading and increasing the percent of fat in enteral feeds. The detected interrelation between substrate oxidation and ventilation outcome became the base of several research to determine the appropriate composition of the nonprotein calories of diet in ventilated patients.

Purpose: We aimed to assess the effect of high-fat dietary modification and nutritional status on ventilatory and final outcomes of pediatric intensive care.

Methods: Fifty-one ventilated children (1 month to 12 years of age) with pulmonary disease who could be enterally fed, in the Cairo University Pediatric intensive care unit, were divided into 2 groups: group A included 25 patients who received isocaloric high-fat, low-carbohydrate diet; group B included 26 patients who received standard isocaloric diet. Comprehensive nutritional assessment was done for all patients.

Results: Group A had a significant reduction in carbon dioxide tension, but no similar reduction in the duration or level of ventilatory support. Assisted minute ventilation was predicted by weight-for-age and caloric intake rather than the type of diet. Poor nutritional status was associated with higher mortality and lower extubation rates. Mild hypertriglyceridemia and some gastrointestinal intolerance were significant in group A, with no impact on the adequacy of energy or protein delivery.

Conclusion: The high-fat enteral feeding protocol may contribute to reducing carbon dioxide tension, with mild hypertriglyceridemia and negligible gastrointestinal intolerance as potential adverse effects. Optimization of nutritional status rather than dietary modification may improve ventilatory and survival outcomes in critically ill-ventilated children.

Key words: Enteral nutrition, High fat diet, Critical illness, Pulmonary disease, Malnutrition

\section{Introduction}

Ventilator dependency constitutes a major problem in the intensive care setting. Many factors are implicated in the pathophysiology of extubation failure, however, malnutrition is considered a major determinant. ${ }^{1)}$ In the 1980s, reports began to appear in the literature describing the effects of nutrition on ventilator settings. Excessive carbohydrate loading in parenteral nutrition was suggested to precipitate respiratory acidosis in adult patients with chronic obstructive pulmonary disease who were unable to adequately compensate for increased carbon dioxide $\left(\mathrm{CO}_{2}\right)$ production. ${ }^{2,3)}$

Thus, attention has been attracted to using nutritional therapy as a means of modulating
Corresponding author: Shrouk Moataz Abdallah, MD Department of Pediatrics, Faculty of Medicine, Cairo University, Cairo University, Giza, Egypt Tel: +201099221393

Fax: +20235729659

E-mail: shroukmoataz14@gmail.com https://orcid.org/0000-0002-2015-757X

Received: 1 August 2018

Revised: 16 February 2019

Accepted: 13 March 2019
Copyright (C) 2019 by The Korean Pediatric Society

This is an open-access article distributed under the terms of the Creative Commons Attribution NonCommercial License (http://creativecommons.org/ licenses/by-nc/4.0/) which permits unrestricted noncommercial use, distribution, and reproduction in any medium, provided the original work is properly cited. 
$\mathrm{CO}_{2}$ production. ${ }^{4)}$ Further studies were done on ventilated intensive care adult patients supporting the use of low carbohydrate-high lipid diets. They showed favorable outcomes in the reduction of $\mathrm{CO}_{2}$ production and the duration of mechanical ventilation (MV) with significant improvement in ventilation settings and respiratory indices. ${ }^{5,6}$ The detected interrelation between substrate oxidation and ventilation outcome became the base of several discussions to determine the appropriate composition of the nonprotein calories of diet in ventilated patients. ${ }^{1)}$

Being a tertiary care center with four busy medical pediatric intensive care units (PICUs), our attention was directed towards finding a new helpful feeding protocol for these patients. Many of critically ill patients experience prolonged ventilation and/or ventilator dependency with distressing financial and social burdens. In addition, ventilator dependency is associated with prolonged length of stay and increased morbidity and mortality. The effect of nutritional status on the outcome of ventilated children has been a point of interest of many pediatric intensive care researchers with no more focusing on the effect of modulating the fat composition of their diets. The present study aimed to test the efficacy of a high-fat feeding protocol in reducing $\mathrm{CO}_{2}$ production, shortening of the duration of $\mathrm{MV}$ and improving final PICU outcomes in pediatric critically ill ventilated patients. The effect of clinical and biochemical nutritional status on all these parameters was tested as a secondary outcome.

\section{Methods}

\section{Patients}

A randomized controlled trial was carried out at the PICUs of Cairo University Children Hospitals, from October 2015 to January 2017. Closed envelopes were used to randomize patients to the type of diet. Identification number for the clinical trial is PACTR20180200 292319. An informed consent form is signed by all parents upon admission to the PICU.

The study included critically ill ventilated children aged one month to 12 years. All patients had at least one acute respiratory problem that necessitated ventilation at any time during PICU stay. Some patients were admitted to the PICU for other reasons than respiratory and then enrolled in the study when they developed a respiratory problem. Patients were recruited when hemodynamic stability was achieved (normal blood pressure and perfusion with no desaturation for 24 hours) and gastrointestinal tract was functioning (no upper or lower gastrointestinal bleeding, no bilious output with normal intestinal sounds). Patients were excluded if they had inborn errors of metabolism or chronic hepatic, renal or endocrinal disorders.

\section{Initial assessment (within 24 hours of recruitment)}

1) Respiratory status

Ventilation and oxygenation status were assessed via arterial blood gases analysis for $\mathrm{PO}_{2}, \mathrm{PCO}_{2}$, and base deficit. Initial MV mode, ventilation settings and lung indices readings were recorded from the digital display of the ventilator. Assisted minute ventilation $(\mathrm{mL} /$ $\mathrm{m}^{2} / \mathrm{min}$ ) was calculated using the recorded expired tidal volume $\left(\mathrm{mL} / \mathrm{m}^{2}\right.$ surface area) multiplied by respiratory rate (breaths $/ \mathrm{min}$ ) at day-1 and -7 of enrollment. It was used as an indicator for the level of ventilatory support delivered to patients.

\section{2) Mortality scoring}

Pediatric index of mortality score 2 (PIM-2) score online calculator was used (www.sfar.org/scores2/pim22.html).

\section{3) Anthropometric measurements}

Weight was recorded using a digital infant scale for patients less than 2 years. For those older than 2 years, the difference between 2 measures was taken: the nursing staff weight with and without carrying the child. The supine length was performed for most patients. For spastic cerebral palsy patients, a predictive equation was used. ${ }^{7)}$ World health organization $z$ score values were calculated for weight-for-age, weight-for-length, length-for-age, body mass index (BMI)-for-age, mid-upper arm circumference (MUAC)-for-age and triceps skinfold-for-age using WHO Anthro (version 3.2.2, January 2011) and macros.

\section{4) Laboratory tests}

Complete blood count with a differential white cell counts, quantitative C-reactive protein and chemistry involving liver and kidney function tests and lipid profile were performed. Prealbumin, as a specific nutritional biomarker, was assayed using immunoturbidimetric assay on chemistry autoanalyzer. ${ }^{8 .}$

\section{Nutritional management}

Patients were divided into 2 groups using sealed envelopes for randomized allocation: Group A, high fat feeding group, included 25 patients who received isocaloric high fat low carbohydrates feeding (30\% carbohydrates, 50\% fats). Fat content was reconstituted to represent 50\% of the nonprotein calories (proteins were not included in energy calculations to be spared for tissue healing, immunity and growth). Group B, standard feeding group, included 26 patients who received standard isocaloric feeding protocol (55\% carbohydrates, 25\% fats).

Predicted resting energy expenditures (PREE) were calculated using the World Health Organization's Equations. ${ }^{9)}$ Target caloric requirements were calculated by multiplication of a stress factor (1.5-2) by the PREE. Protein requirements were determined based on ASPEN 2009 recommendations. ${ }^{10)}$ Feeds were gradually advanced over 6 days.

Infants younger than 4 months of age were fed a ready-made formula with fat representing 50\% of nonprotein calories (Infatrini, Nutricia). For those older than 4 months of age, high-fat enteral feeds 
are formulated by adding olive oil to blended food (Supplementary material), after revising the national mimeographic data of Food Composition Tables for Egypt, Ministry of Health and Population, General Organization for Teaching Hospitals and Institutes, National Nutrition Institute, 2nd edition, May 2006.

\section{Monitoring}

All clinical parameters related to the respiratory system (ventilator settings, lung indices and arterial blood gases) at day 7 of recruitment. All basic laboratory tests and prealbumin were repeated at day 7 of recruitment. Daily record of feeding advancement and interruption was done with documentation of the reasons for interruptions. Percentage of target calories and proteins achieved at day 7 of recruitment was recorded.

\section{Outcome parameters}

The effect of dietary modification as a primary outcome on: extubation rate, carbon dioxide tension $\left(\mathrm{pCO}_{2}\right)$ and $\mathrm{pH}$, assisted minute ventilation, biochemical markers, and mortality.

\section{The effect of nutritional status on the previous parameters was tested as a secondary outcome: data management}

Clinical and laboratory data were analyzed using SPSS ver. 16 (SPSS Inc., Chicago, IL, USA). In variables that were normally distributed, mean and standard deviation were performed and chi-square test was used for analysis of nonparametric data. Median and interquartile range were obtained for variables that were not normally distributed. Statistical significance is considered when $P$ value is less than or equals to 0.05. Difference between outcome groups was assessed using the 1-way analysis of variance (ANOVA) with Fisher Least Significant Difference used as post hoc test. Multistep regression analyses were performed to determine the predicting variables for outcome parameters. Multistep regression analysis and multivariate ANOVA (MANOVA) were used to determine multiple dependent factors affecting day-7, -14, and final outcomes.

\section{Ethical committee approval}

The study was approved by the ethical committee of the Pediatrics department, Faculty of Medicine, Cairo University (approval number: I-050314).

\section{Results}

The study included 51 critically ill children fulfilling the inclusion criteria, divided according to the type of diet into 2 groups as previously detailed. Thirty-five patients (68.6\%) were enrolled within 24-48 hours of admission (early enrollment group) and the rest of patients were enrolled after more than 48 hours (late enrollment group) with no significant difference between groups. Demographic characteristics of the included patients are shown in Table 1.

Both study groups were homogenous as regards age, gender and PIM-2 scoring. All patients had at least one acute respiratory problem; pneumonia was the most frequent presenting diagnosis $(\mathrm{n}=47,92.2 \%)$, some patients had additional chronic upper or lower respiratory problems. Forty patients (78.4\%) had associated nonrespiratory diagnostic categories including several neurological, cardiac, musculoskeletal and immunological diseases. Neurological diseases were the most frequent associated conditions ( $n=25,49 \%)$ with statistically significant higher frequencies among group $B$ $\left(P=0.003^{*}\right)$ (Table 1$)$.

Malnutrition was highly prevalent among both groups (defined as World Health Organization $z$ scores $\leq-2$ standard deviation [SD]) in terms of the following parameters: weight-for-age ( $n=35,68.6 \%)$, weight-for-length $(n=32,62.7 \%)$ and BMI-for-age $(n=31,60.8 \%)$ with no significant differences. Severe malnutrition (defined as $Z$ scores $\leq-3 \mathrm{SD}$ ) was more frequent among group B with statistically significant differences in terms of weight-for-age, weight-for-length,

Table 1. Demographic characteristics of the study groups

\begin{tabular}{|c|c|c|c|c|}
\hline Characteristic & All $(n=51)$ & Group A ( $n=25)$ & Group B $(n=26)$ & $P$ value \\
\hline Age (mo) & $12(3.7-31.5)$ & $12(3.7-51)$ & $10(3-24)$ & 0.93 \\
\hline \multicolumn{5}{|l|}{ Sex } \\
\hline Male & $32(62.7)$ & $16(64)$ & $16(61.5)$ & 0.85 \\
\hline Female & 19 (37.3) & $9(36)$ & $10(38.5)$ & \\
\hline \multicolumn{5}{|l|}{ Enrollment groups } \\
\hline Early (within 48 hr) & 35 (68.6) & $20(80)$ & $15(57.7)$ & 0.08 \\
\hline Late (>48 hr) & $16(31.4)$ & $5(20)$ & $11(42.3)$ & \\
\hline PIM-2 & 13.5 (5.3-28.2) & $10.2(5.75-27.7)$ & $17.1(5.2-28.8)$ & 0.27 \\
\hline \multicolumn{5}{|c|}{ Acute respiratory condition } \\
\hline Pneumonia & 47 (92.1) & $21(84)$ & $26(100)$ & 0.20 \\
\hline ARDS & $3(5.9)$ & $3(12)$ & $0(0)$ & \\
\hline Status asthmaticus & $1(2)$ & $1(4)$ & $0(0)$ & \\
\hline
\end{tabular}


Table 1. Demographic characteristics of the study groups (Continued)

\begin{tabular}{|c|c|c|c|c|}
\hline Characteristic & All $(n=51)$ & Group A (n=25) & Group B $(n=26)$ & $P$ value \\
\hline Chronic respiratory condition & $10(19.6)$ & $6(24)$ & $4(15.4)$ & 0.66 \\
\hline \multicolumn{5}{|l|}{ Upper airway } \\
\hline Laryngomalacia & $2(3.9)$ & $0(0)$ & $2(7.7)$ & \\
\hline Adenoid hypertrophy & $1(2)$ & $1(4)$ & $0(0)$ & \\
\hline \multicolumn{5}{|l|}{ Lower airway } \\
\hline Undiagnosed & $3(5.9)$ & $3(12)$ & $0(0)$ & \\
\hline Persistent asthma & $1(2)$ & $1(4)$ & $0(0)$ & \\
\hline Cystic fibrosis & 1(2) & $1(4)$ & $0(0)$ & \\
\hline Tuberculous lung fibrosis & $1(2)$ & $0(0)$ & $1(3.8)$ & \\
\hline BPD & $1(2)$ & $0(0)$ & $1(3.8)$ & \\
\hline Neurological disorder & $25(49)$ & $5(20)$ & $20(77)$ & 0.003 \\
\hline \multicolumn{5}{|l|}{ Acute } \\
\hline Convulsions & $4(7.8)$ & $1(4)$ & $3(11.5)$ & \\
\hline Intracranial hemorrhage & $2(4)$ & $0(0)$ & $2(7.7)$ & \\
\hline Toxic encephalopathy & $1(2)$ & $1(4)$ & $0(0)$ & \\
\hline CNS infection & $1(2)$ & $0(0)$ & $1(3.8)$ & \\
\hline \multicolumn{5}{|l|}{ Chronic } \\
\hline Hydrocephalus & $5(9.8)$ & $1(4)$ & $4(15.4)$ & \\
\hline Cerebral palsy & $3(5.8)$ & $2(8)$ & $1(3.8)$ & \\
\hline Neurodegenerative disease & $4(7.8)$ & $1(4)$ & $3(12)$ & \\
\hline Cystic encephalomalacia & $1(2)$ & $0(0)$ & $1(3.8)$ & \\
\hline GBS & $3(5.9)$ & $0(0)$ & $3(11.5)$ & \\
\hline Pompe disease & $1(2)$ & $0(0)$ & $1(3.8)$ & \\
\hline Cardiac diagnosis & $10(25)$ & $3(20)$ & $7(26.9)$ & \\
\hline Congenital heart disease & & & & 0.60 \\
\hline Cyanotic & $3(7.5)$ & $0(0)$ & & \\
\hline Acyanotic & $5(12.5)$ & $2(13.3)$ & & \\
\hline \multicolumn{5}{|l|}{ Acquired heart disease } \\
\hline DCM & $1(2.5)$ & $0(0)$ & $1(4)$ & \\
\hline Kawasaki & $1(2.5)$ & $1(6.7)$ & $0(0)$ & \\
\hline Immunological (SCID) & $1(2)$ & $1(4)$ & $0(0)$ & 0.66 \\
\hline
\end{tabular}

Values are presented as median (interquartile range) or number (\%).

Group A, high fat feeding group; group B, standard feeding group; PIM-2, pediatric index of mortality score 2; ARDS, acute respiratory distress syndrome; BPD, bronchopulmonary dysplasia; CNS, central nervous system; GBS, Guillian Barre syndrome; DCM, dilated cardiomyopathy; SCID, severe combined immunodeficiency. Boldface indicates a statistically significant difference with $P<0.05$.

and BMI-for-age. No similar difference between both groups was noted in terms of MUAC-for-age (Table 2).

Feeding was initiated within 24-48 hours of admission. Several avoidable and nonavoidable causes of feeding interruption were recorded, such as severe respiratory distress, gastrointestinal bleeding, paralytic ileus, poor adherence of PICU staff to feeding regimens were recorded. Higher frequency of interruptions due to gastrointestinal intolerances was recorded among the high-fat feeding group with no statistically significant impact on the percent of energy and proteins achieved at day 7 (Table 2).

\section{MV outcomes}

Patients were ventilated for a median of 7 days and a range of 311 days for group $A$ and a median of 5 days and a range of 4-7 days for group B with no significant difference in the duration of MV according to the type of diet. Synchronized intermittent mandatory ventilation (SIMV) was the most commonly used initial ventilation mode with no difference between the groups A and B. Both groups showed a significant change in ventilation modes before and after the intervention, Table 3.

Fifteen patients (29.4\%) were extubated at day 7 with no statistically significant difference between both groups. Significantly lower extubation rates at day 14 were detected among group B with no 
Table 2. The prevalence of malnutrition among the study groups

\begin{tabular}{lccrc}
\hline Malnutrition prevalence & $\begin{array}{c}\text { All } \\
(n=51)\end{array}$ & $\begin{array}{c}\text { Group A } \\
(\mathrm{n}=25)\end{array}$ & $\begin{array}{c}\text { Group B } \\
(\mathrm{n}=26)\end{array}$ & $P$ value \\
\hline Weight/age (z Score) & & & & \\
More than -2 SD & $11(21.6)$ & $6(24)$ & $5(19.2)$ & \\
-2 to -3 SD & $16(31.4)$ & $11(44)$ & $5(19.2)$ & 0.01 \\
Less than -3 SD & $19(37.3)$ & $4(16)$ & $15(57.7)$ & \\
Weight/length (z score) & & & & \\
More than -2 SD & $14(27.5)$ & $8(32)$ & $6(23.1)$ & \\
-2 to -3 SD & $12(23.5)$ & $8(32)$ & $4(15.4)$ & 0.04 \\
Less than -3 SD & $20(39.2)$ & $5(9.8)$ & $15(57.7)$ & \\
BMI/age (z Score) & & & & \\
More than -2 SD & $15(29.4)$ & $8(32)$ & $7(26.9)$ & \\
-2 to -3 SD & $11(21.6)$ & $8(32)$ & $3(11.5)$ & 0.03 \\
Less than -3 SD & $20(39.2)$ & $5(9.8)$ & $15(57.7)$ & \\
MUAC/age (z Score) & & & & \\
More than -2 SD & $15(29.4)$ & $9(36)$ & $6(23.1)$ & \\
-2 to -3 SD & $6(11.7)$ & $1(4)$ & $5(19.2)$ & 0.18 \\
Less than -3 SD & $17(33.3)$ & $7(28)$ & $10(38.5)$ &
\end{tabular}

Values are presented as number (\%).

Group A, high fat feeding group; group B, standard feeding group; SD, standard deviation; BMl, body mass index; MUAC, mid-upper arm circumference.

Boldface indicates a statistically significant difference with $P<0.05$.

Table 3. Outcome parameters of the study groups

\begin{tabular}{lccc}
\hline Outcome parameter & Group A (n=25) & Group B $(\mathrm{n}=26)$ & $P$ value \\
\hline MV mode & & & \\
Day 1 & & & \\
SIMV & $21(84)$ & $1(3.8)$ & \\
HFOV & $2(8)$ & 0.31 \\
PRVC & $2(8)$ & $1(3.8)$ & \\
ET-CPAP & $0(0)$ & & \\
Day 7 & & $9(34.6)$ & 0.81 \\
SIMV & $10(40)$ & $1(3.8)$ & \\
HFOV & $1(4)$ & $0(0)$ & \\
PRVC & $1(4)$ & $1(3.8)$ & \\
PSV & $0(0)$ & $3(11.5)$ & \\
ET-CPAP & $2(8)$ & 0.000 & \\
$P$ value & 0.001 & &
\end{tabular}

significant correlation to the type of $\operatorname{diet}(\beta=0.378, P=0.22)$. The day14 outcome showed a significant correlation to weight for age $(\beta=$ -0.633, $P=0.01$ ), therefore, the lower extubation rates for group $\mathrm{B}$ at day 14 may be attributed to the higher prevalence of severe malnutrition among this group as previously discussed. Several anthropometric and laboratory parameters showed significant differences in multiple group comparison for day-14 outcome (Table 4).

A higher level of ventilatory support was needed for group B at day 7 as evident by a 2-fold increase in assisted minute ventilation, however, the difference was not statistically significant (Table 3).
Table 3. Outcome parameters of the study groups (contiuned)

\begin{tabular}{|c|c|c|c|}
\hline Outcome parameter & Group A ( $n=25)$ & Group B (n=26) & $P$ value ${ }^{\mathrm{a}}$ \\
\hline \multicolumn{4}{|c|}{ Assisted minute ventilation ( $\left.\mathrm{L} / \mathrm{m}^{2} / \mathrm{min}\right)$} \\
\hline Day 1 & $5.86(3.6-8.9)$ & $4.52(3.3-6.9)$ & 0.23 \\
\hline Day 7 & $6.19(4.7-7.8)$ & $8.17(4.5-10.8)$ & 0.36 \\
\hline$P$ value $^{\mathrm{b})}$ & 0.08 & 0.74 & \\
\hline \multicolumn{4}{|l|}{$\mathrm{pH}$} \\
\hline Day 1 & $7.37 \pm 0.1$ & $7.46 \pm 0.1$ & 0.06 \\
\hline Day 7 & $7.46 \pm 0.1$ & $7.45 \pm 0.1$ & 0.71 \\
\hline$P$ value $e^{\text {b) }}$ & 0.004 & 0.81 & \\
\hline \multicolumn{4}{|l|}{$\mathrm{pCO}_{2}$} \\
\hline Day 1 & $47 \pm 15.1$ & $42.24 \pm 15.9$ & 0.11 \\
\hline Day 7 & $37.98 \pm 8.3$ & $42.14 \pm 13.4$ & 0.22 \\
\hline$P$ value $e^{\text {b) }}$ & 0.006 & 0.97 & \\
\hline \multicolumn{4}{|l|}{ Albumin (g/dL) } \\
\hline Day 1 & $3.4(2.8-3.7)$ & $3.65(3.1-4.3)$ & 0.04 \\
\hline Day 7 & $3.1(2.8-3.5)$ & $3.45(2.7-3.8)$ & 0.07 \\
\hline$P$ value $e^{\text {b) }}$ & 0.11 & 0.004 & \\
\hline
\end{tabular}

Prealbumin $(\mathrm{mg} / \mathrm{dL})$

$\begin{array}{lccc}\text { Day 1 } & 8.25(4.5-11.5) & 11.05(7.2-13.4) & 0.23 \\ \text { Day 7 } & 11.75(8.7-20.7) & 15.1(11.4-19.2) & 0.52 \\ P \text { value }^{\text {b) }} & 0.002 & 0.19 & \\ \text { Triglycerides (mg/dL) } & & & \\ \text { Day 1 } & 165(74-232) & 150(127.3-199.3) & 0.95 \\ \text { Day 7 } & 216.5(173.8-346) & 158(151-257) & 0.11 \\ P_{\text {value }}^{\text {b) }} & 0.005 & 0.41 & \end{array}$

Nutritional facts at day 7

\begin{tabular}{lccc} 
\% proteins achieved & $50(48.8-75)$ & $50(33.3-100)$ & 0.62 \\
\% calories achieved & $60(40-83)$ & $49.5(23.8-100)$ & 0.75 \\
Day-7 outcomes & & & \\
Extubated & $8(32)$ & $7(27)$ & \\
Not extubated & $15(60)$ & $15(57.6)$ & 0.70 \\
Dead & $2(8)$ & $4(15.4)$ & \\
Day-14 outcome & & & \\
Extubated & $9(36)$ & $2(7.6)$ & $0.03^{*}$ \\
Not extubated & $10(40)$ & $12(46)$ & \\
Dead & $4(16)$ & $10(38.4)$ & \\
Final outcome & & & \\
Death & $12(48)$ & $18(69.2)$ & 0.16 \\
Discharge & $11(44)$ & $5(19.2)$ & \\
Admitted & $2(8)$ & $3(11.5)$ & \\
\hline
\end{tabular}

Values are presented as number (\%), median (interquartile range), or mean \pm standard deviation.

Group A, high fat feeding group; group B, standard feeding group; MV, mechanical ventilation; SIMV, synchronized intermittent mandatory ventilation; HOFV, high-frequency oscillatory ventilation; PRVC, pressure regulated volume control; PSV, pressure support ventilation; ET-CPAP, endotracheal continuous positive airway pressure.

${ }^{\text {a) }} P$ value for differences between the groups $A$ and $B .{ }^{b} P$ value for differences before and after the intervention (day 1 and day 7 of enrollment) for each group.

Boldface indicates a statistically significant difference with $P<0.05$. 
Table 4. Multiple group comparisons for day-14 outcome

\begin{tabular}{|c|c|c|c|c|c|}
\hline Parameter & Extubated & Not extubated & Dead & $P$ value $^{\text {a) }}$ & $P$ value \\
\hline \multicolumn{6}{|l|}{$z$ scores (day 1) } \\
\hline Weight for length & $-0.7(-2.1$ to 0.4$)$ & $-2.7(-4.8$ to -0.4$)$ & $-2.9(-5.5$ to -2.2$)$ & & 0.02 \\
\hline Weight for age & $-1.4(-2.5$ to -1$)$ & $-2.9(-4.8$ to -2.5$)$ & $-3.6(-4.6$ to -2.6$)$ & 0.03 & 0.03 \\
\hline BMl for age & $-0.6(-2.1$ to 0.5$)$ & $-3.7(-5.1$ to 0.4$)$ & $-2.8(-5.6$ to -2.1$)$ & 0.04 & 0.03 \\
\hline PIM-2 & $6.2(4.2-11.4)$ & $15.7(4.7-30.6)$ & $27.1(19.4-34.75)$ & & 0.00 \\
\hline \multicolumn{6}{|l|}{ Albumin } \\
\hline Day 1 & $3.7(3.1-4)$ & $3.5(2.9-4)$ & $3(2.5-3.4)$ & & 0.03 \\
\hline Day 7 & $3.6(2.9-3.9)$ & $3.2(2.6-3.7)$ & $28(1.9-3.5)$ & & 0.02 \\
\hline \multicolumn{6}{|l|}{ Prealbumin } \\
\hline Day 1 & $10.1(7.3-11.8)$ & $10(7.1-14.4)$ & $6.4(3.7-11.3)$ & & 0.04 \\
\hline Day 7 & $20.5(9.2-23.8)$ & $14.6(10.1-18.7)$ & $8.5(2.1-9.4)$ & & 0.01 \\
\hline
\end{tabular}

Values are presented as median (interquartile range).

$\mathrm{BMI}$, body mass index, PIM-2, pediatric index of mortality score 2 .

a) $P$ value comparing the patients who were extubated to those who were dead at day-14 of enrollment. ${ }^{\text {b) }} P$ value comparing the patients who were still ventilated to those who were dead at day-14 of enrollment.

Boldface indicates a statistically significant difference with $P<0.05$.
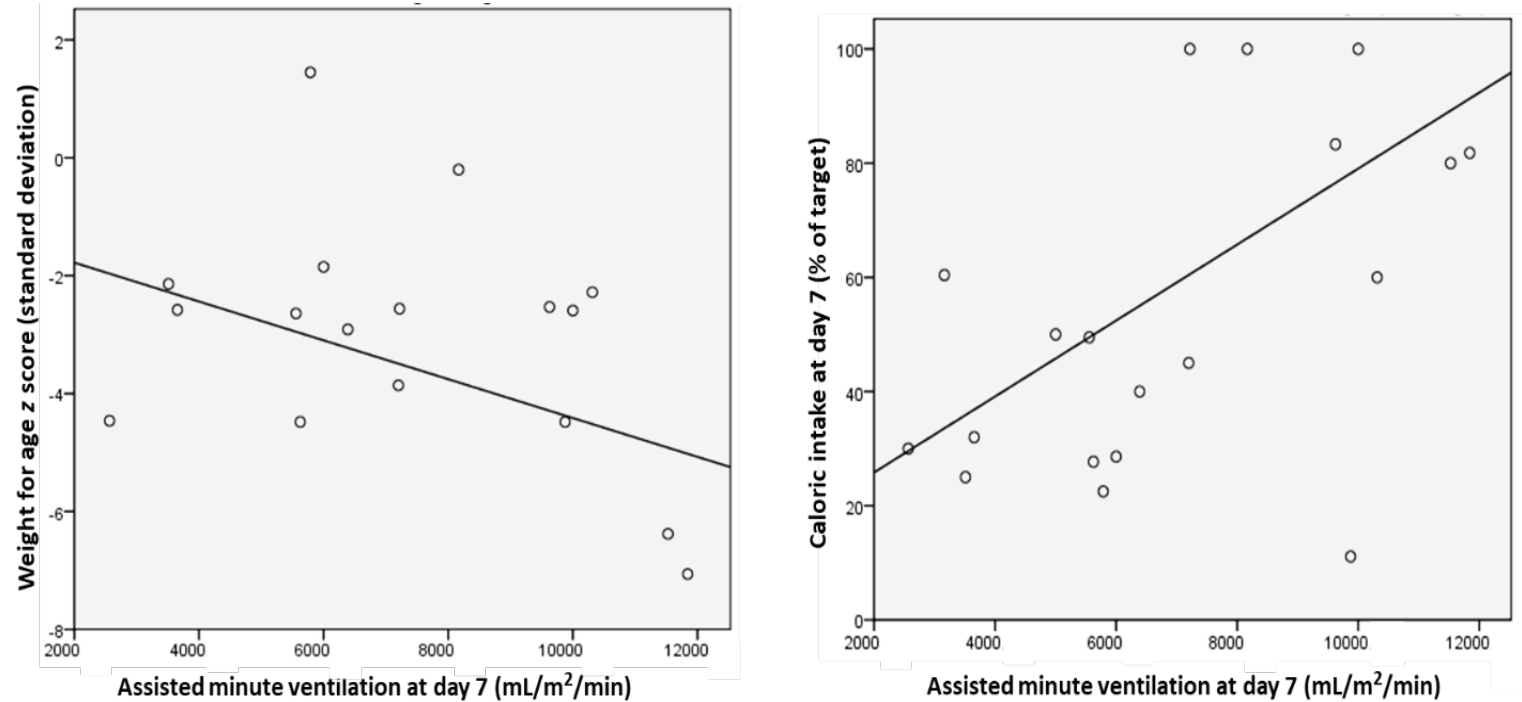

Fig. 1. Weight-for-age and caloric intake as predictors of assisted minute ventilation at day 7 .

Assisted minute ventilation was significantly predicted by weight for age $(\beta=-0.485, P=0.04)$ and caloric intake $(\beta=0.66, P=0.001)$, rather than the type of diet (Fig. 1).

\section{Laboratory outcomes}

There were no significant differences between the groups A and $B$ in the initial blood gases, blood counts, routine chemistry, prealbumin, and lipid profiles. Stepwise regression analysis revealed a statistically significant difference in the laboratory outcome parameters based on the type of diet given to the patients $(F=3.72, P=$ 0.006 , Wilk's $\beta=0.446$, partial $\eta^{2}=0.55$ ). High-fat feeding group showed a significant $\mathrm{pH}$ rise $\left(F=8.21, P=0.007\right.$, partial $\left.\eta^{2}=0.21\right)$ and $\mathrm{pCO}_{2}$ reduction after the intervention compared to nonsignificant changes among group B. Significant improvement of prealbumin and elevation of triglyceride levels ( $F=5.08, P=0.03$, partial $\eta^{2}=0.14$ ) were detected among group A at day 7 of enrollment (Table 3). No similar difference was noted according to the presence of neurological diseases ( $F=1.02, P=0.44$, Wilk's $\beta=0.746$, partial $\eta^{2}=0.25$ ).

\section{Mortality}

Thirty patients (58.8\%) were dead by the end of the study period with 5 patients were still admitted to the intensive care unit due to ventilator dependency. No significant differences were found in day7 mortality and overall mortality rates between the groups A and B. The mortality group showed significantly lower anthropometric $Z$ score values and biochemical markers with higher mortality scoring 
(Tables 4, 5). Significantly higher group B mortality rates at day 14 were detected ( $P=0.03$ ) (Table 3$)$, could be attributed to higher prevalence of severe malnutrition among this group.

Final outcome was significantly predicted by weight for age $(\beta=$ $-5.725, P=0.001)$, BMI for age $(\beta=3.95, P=0.004)$, and MUAC for age $(\beta=0.77, P=0.005)$, with no effect of the dietary modification $(\beta=$ $0.002, P=0.98)$ or the presence of neurological diagnosis $(\beta=0.136$, $P=0.35$ ). The overall model fit was $R^{2}=0.543, P=0.003$. With stepwise regression analysis, all variables were excluded and MUAC for age was the strongest predicting variable $(\beta=0.467, P=0.001)$ (Fig. 2).

\section{Discussion}

The high-fat diet was reported to reduce $\mathrm{CO}_{2}$ and improve $\mathrm{pH}$ values and this was a significant result shared between the current study and some previous adult studies on ventilated patients with

Table 5. Multiple group comparisons for the final outcome

\begin{tabular}{lrcc}
\hline Parameter & \multicolumn{1}{c}{ Dead } & Discharged & $P$ value \\
\hline Anthropometry at day 1 (z score) & & & \\
Weight for length & $-3.4(-5.2$ to -2.1$)$ & $-1(-3.2$ to 0.2$)$ & 0.03 \\
Weight for age & $-3.7(-5.6$ to -2.6$)$ & $-1.5(-3.3$ to -1$)$ & 0.02 \\
MUAC for age & $-4(-5.1$ to -1.5$)$ & $-0.2(-3.2$ to 0.7$)$ & 0.01 \\
BMl for age & $-3.9(-6.1$ to -2.3$)$ & $-1(-3.2$ to 0.3$)$ & 0.03 \\
Mortality scoring & & & \\
$\begin{array}{l}\text { PIM-2 (\%) } \\
\text { Laboratory parameters }\end{array}$ & $21.9(10.2-31)$ & $8.4(5.2-13.5)$ & 0.03 \\
Prealbumin day 7 (mg/dL) & $11(8.5-15.8)$ & $16.3(10.6-23.1)$ & 0.04 \\
\hline
\end{tabular}

Values are presented as median (interquartile range).

MUAC, midupper arm circumference; BMI, body mass index; PIM-2, pediatric index of mortality score 2.

Boldface indicates a statistically significant difference with $P<0.05$.

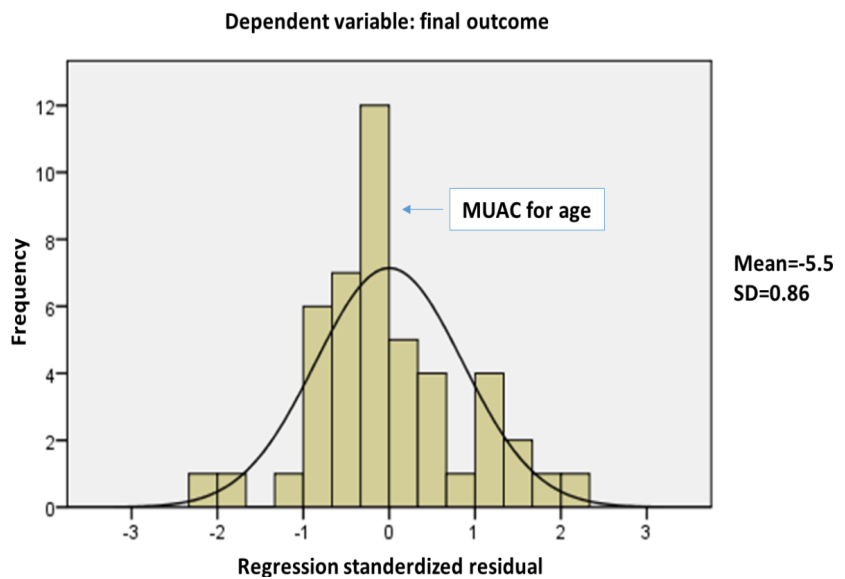

Fig. 2. Stepwise regression analysis showing mid-upper arm circumference (MUAC)-for-age as the strongest predictor of the final outcome. SD, standard deviation. respiratory diseases. ${ }^{5,6)}$ However, spot measurement of $\mathrm{pH}$ and $\mathrm{CO}_{2}$ cannot be used as a sole indicator of diet efficacy. This $\mathrm{CO}_{2}$ reduction was associated with a similar significant reduction in the level of ventilatory support needed for those patients, ${ }^{5,6)}$ unlike the current study; it did not induce a similar effect on assisted minute ventilation used as an indicator for the level of ventilatory support.

Many studies found that the caloric intake rather than the amount of fat or carbohydrate ingested was the predictor of hypercapnia, ${ }^{11}$ in the current study, caloric intake was a significant predictor for assisted minute ventilation rather than the type of diet. The total duration of ventilation was reduced by high fat dietary modification in some reports, ${ }^{5,6)}$ while in the current study and other literature reports, it did not seem to induce a similar effect. ${ }^{12)}$

To our knowledge, pediatric studies about the effect of the highfat dietary modification on mechanically ventilated patients were lacking till the time of manuscript writing. This makes referring to the previously mentioned adult studies is not very ideal due to different physiology and age-specific pulmonary diseases.

Ventilation outcomes in the current study were rather affected by nutritional status. This could be justified by better anthropometric $Z$ score values for extubated patients and weight for age as a predictor for the level of ventilatory support. Similar results were previously reported by Grippa et al. ${ }^{13)}$ where the duration of MV was significantly predicted by weight for age and upper arm muscle area for age. Many studies reported that underweight children according to BMI $z$ scores required prolonged ventilatory support. ${ }^{14,15)}$ Albumin and prealbumin levels were significantly higher among extubated patients, however, they were not predictors for the outcomes of MV, similar to what is reported by Grippa et al. ${ }^{13)}$

Improvement of prealbumin levels in the high-fat group cannot be justified with the available data because of achieving similar caloric and protein intake at the time of follow up by both groups. It would have been better explained if bio-electrical impedance was available for body composition analysis. Lean tissue index would have particular importance due to its positive correlation to prealbumin level. ${ }^{16)}$ Also, further research is required to assess the effect of high-fat diet on protein metabolism in critically ill children.

Mortality was predicted by the nutritional status as reported by Nourmohammadi et al. ${ }^{17)}$ rather than the effect of high-fat diet. Several anthropometric parameters were significantly different between survivors and non-survivors similar to the report of Bechard et al. ${ }^{14)}$ Prealbumin concentrations were found to be significantly higher in patients who survived to discharge, however, showed no consistent association to final PICU outcomes. ${ }^{18)}$

We faced some limitations in the current study which were similar to Grippa et al. ${ }^{13)}$ report in many aspects. Both are single-center studies and cannot be extended to other PICUs with different characteristics. During the study period, there were many patients with associated nonrespiratory conditions. This might have interfered with prompt evaluation of diet efficacy on the respiratory system 
alone. Many patients with high-risk diagnoses according to PIM-2 scoring were enrolled, added to the high prevalence of malnutrition among the study groups which was nearly 3 times higher compared to the report of Grippa et al. ${ }^{13)}$ This may have contributed to the observed high mortality during the study period than the average mortality of PICUs. Self-funding limited the nutritional markers measured and lead to lack of continuous $\mathrm{CO}_{2}$ tracking. Taking the anthropometric measurements was challenging especially in edematous patients. Additionally, in some neurological cases the height was calculated by predictive equations ${ }^{7)}$ which may not be very accurate.

To conclude, high-fat enteral feeding protocol may contribute to the reduction of $\mathrm{PCO}_{2}$ with no major impact on ventilatory and final PICU outcomes. Optimization of nutritional status has a major role in improving ventilatory and survival outcomes in critically ill ventilated children. Further advanced multicentric clinical trials are required in pediatric critically ill ventilated patients with respiratory diseases to determine the optimal macronutrient composition of their enteral feeds.

\section{Conflicts of interest}

No potential conflict of interest relevant to this article was reported.

\section{Acknowledgments}

I would like to thank all the PICU team, the laboratory team and all my patients' caregivers for their cooperation during the study period. Special thanks go to Dr. Radwa Marawan Abdel Halim, lecturer of Chemical Pathology, Cairo University for her sincere cooperation in facilitating all the laboratory procedures.

\section{Supplementary material}

Supplementary data can be found via https://doi.org/10.3345/ kjp.2018.06835.

\section{References}

1. Esquinas AM, editor. Noninvasive mechanical ventilation and difficult weaning in critical care; key topics and practical approaches. Cham
(Switzerland): Springer International Publishing, 2016:46.

2. Askanazi J, Rosenbaum SH, Hyman AI, Silverberg PA, Milic-Emili J, Kinney JM. Respiratory changes induced by the large glucose loads of total parenteral nutrition. JAMA 1980;243:1444-7.

3. Covelli HD, Black JW, Olsen MS, Beekman JF. Respiratory failure precipitated by high carbohydrate loads. Ann Intern Med 1981;95:57981.

4. Iapichino G. Nutrition in respiratory failure. Intensive Care Med 1989; 15:483-5.

5. al-Saady NM, Blackmore CM, Bennett ED. High fat, low carbohydrate, enteral feeding lowers $\mathrm{PaCO} 2$ and reduces the period of ventilation in artificially ventilated patients. Intensive Care Med 1989;15:290-5.

6. Faramawy MA, Abd Allah A, El Batrawy S, Amer H. Impact of high fat low carbohydrate enteral feeding on weaning from mechanical ventilation. Egypt J Chest Dis Tuberc 2014;63:931-8.

7. Chumlea WC, Guo SS, Steinbaugh ML. Prediction of stature from knee height for black and white adults and children with application to mobility-impaired or handicapped persons. J Am Diet Assoc 1994; 94:1385-8, 1391.

8. Shahangian S, Agee KA, Dickinson RP. Concentration dependencies of immunoturbidimetric dose-response curves: immunoturbidimetric titer and reactivity, and relevance to design of turbidimetric immunoassays. Clin Chem 1992;38:831-40.

9. Food and Agriculture Organization. Energy and protein requirements: report of a joint FAO/WHO/UNU expert consultation. World Health Organization Technical Report Series 724. Geneva (Switzerland): World Health Organization, 1985.

10. Mehta NM, Compher C; A.S.P.E.N. Board of Directors. A.S.P.E.N. Clinical guidelines: nutrition support of the critically ill child. JPEN J Parenter Enteral Nutr 2009;33:260-76.

11. Talpers SS, Romberger DJ, Bunce SB, Pingleton SK. Nutritionally associated increased carbon dioxide production. Excess total calories vs high proportion of carbohydrate calories. Chest 1992;102:551-5.

12. van den Berg B, Bogaard JM, Hop WC. High fat, low carbohydrate, enteral feeding in patients weaning from the ventilator. Intensive Care Med 1994;20:470-5.

13. Grippa RB, Silva PS, Barbosa E, Bresolin NL, Mehta NM, Moreno YM. Nutritional status as a predictor of duration of mechanical ventilation in critically ill children. Nutrition 2017;33:91-5.

14. Bechard LJ, Duggan C, Touger-Decker R, Parrott JS, Rothpletz-Puglia $\mathrm{P}$, Byham-Gray L, et al. Nutritional status based on body mass index is associated with morbidity and mortality in mechanically ventilated critically Ill children in the PICU. Crit Care Med 2016;44:1530-7.

15. Chaitra KM, Bhavya G, Harish S, Patel S, Anjum SK. Influence of nutritional status on clinical outcomes in critically ill children. Int J Contemp Pediatr 2018;5:462-6.

16. Aatif T, Hassani K, Alayoud A, Maoujoud O, Ahid S, Benyahia M, et al. Parameters to assess nutritional status in a Moroccan hemodialysis cohort. Arab J Nephrol Transplant 2013;6:89-97.

17. Nourmohammadi M, Moghadam OM, Lahiji MN, Hatamian S, Shariatpanahi ZV. Effect of fat-based versus carbohydrate-based enteral feeding on glycemic control in critically Ill patients: a randomized clinical trial. Indian J Crit Care Med 2017;21:500-5.

18. Ong C, Han WM, Wong JJ, Lee JH. Nutrition biomarkers and clinical outcomes in critically ill children: a critical appraisal of the literature. Clin Nutr 2014;33:191-7. 